\title{
ADENOME PLEOMORPHE A LOCALISATION EXTRA-PAROTIDIENNE
}

\author{
E. GASSAB, A. BERKAOUI, S. KEDOUS, A. KORBI, N. KHAIREDDINE, K. HARRATHI , \\ A. MOUSSA* A . RZIG**, F. EL KADHI, J. KOUBAA, A. GASSAB \\ SERVICE D'ORL ET CHIRURGIE CERVICAUX FACIALE. \\ * SERVICE D'ANATOMIE ET CYTOLOGIE PATHOLOGIQUE \\ ** SERVICE D'IMAGERIE MÉDICALE \\ HÔPITAL FATTOUMA BOURGUIBA MONASTIR
}

\begin{abstract}
OBJECTIFS : L'adénome pléomorphe est une tumeur bénigne hétérogène des glandes salivaires. Ses localisations extra-parotidiennes sont rares. L'objectif de notre étude est d'en décrire les particularités épidémiologiques, cliniques et thérapeutiques.

Matériel et Méthodes : A travers une étude rétrospective menée sur une période de 10 ans (2000-2009), nous avons colligé 15 cas d'adénome pléomorphe extra-parotidien soit 34,8\% des adénomes pléomorphes.

Résultats : Les tumeurs siégeaient au niveau de la glande submandibulaire dans $40 \%$ des cas, du palais dans $33 \%$ des cas, de la lèvre supérieure dans $20 \%$ des cas et de l'espace para-pharyngé dans $7 \%$ des cas. L'âge moyen était de 48 ans avec une prédominance féminine. Les tumeurs évoluaient depuis 4 ans en moyenne. Tous les patients ont été opérés. Aucune récidive ni transformation maligne n'ont été rapportées avec un recul moyen de un an.

Conclusion : Les localisations extra-parotidiennes de l'adénome pléomorphe sont dominées par la glande submandibulaire et les glandes salivaires accessoires du palais et des lèvres. Chaque localisation se distingue par ses particularités cliniques et thérapeutiques.
\end{abstract}

Mots clés : Adénome pléomorphe, glande submandibulaire, glande salivaire accessoire

Objectives: : Pleormorphic adenoma is a benign tumor of salivary gland. It mainly occurs in the parotid gland. The submandibular and minor salivary glands are rarely sites of occurrence. We describe the features of pleomorphic adenoma occurring at these sites.

Material and methods: Between 2000 and 2009, 15 cases of pleomorphic adenoma occurring externly to the parotid have been collected.

Results: Tumors were seen in the submandibular gland in $40 \%$ of cases, in the hard palate in $33 \%$ of cases, in the upper lip in $20 \%$ of cases and in the parapharyngeal space in $7 \%$ of cases. The mean age of patients was 48 years. The majority of cases were female. All patients were operated. We didn't report recurrence or malignants tumors after one year follow-up.

Conclusion: After the parotid gland, the most common site of a pleomorphic adenoma is the submandibular gland followed by minor salivary gland of palate and lips. Each localisation has his clinical and therapeutic particularities.

Key words : pleomorphic adenoma, submandibular gland, minor salivary gland

\section{INTRODUCTION}

L'adénome pléomorphe est la tumeur la plus fréquente des glandes salivaires $(1,2,3)$. Ses localisations extraparotidiennes sont rares $(1,2)$. Elles se distinguent de la parotide par leurs caractéristiques cliniques, histologiques et thérapeutiques $(2,4,5)$. Elles sont réparties entre la glande submandibulaire et les glandes salivaires accessoires. Le but de cette étude est de décrire les aspects de l'adénome pléomorphe dans ses différentes localisations extraparotidiennes.

\section{PATIENTS ET METHODES}

C'est une étude rétrospective portée sur dix ans (20002009), ayant colligée 15 cas d'adénome pléomorphe à localisation extra-parotidienne parmi un ensemble de 43 cas, soit $34,8 \%$.

II a été précisé les antécédents pathologiques des patients, les symptômes les ayant amenés à consulter et les données de l'examen clinique. Cinq patients ont béné ficié d'une échographie cervico-faciale, 6 patients d'une tomodensitométrie et 2 patients d'une imagerie par réso- 
nance magnétique. Le diagnostic histologique pré-opératoire s'est basé sur la cytoponction dans 5 cas et sur la biopsie tumorale dans 2 cas. Tous les patients ont été opérés. L'évolution a été précisée pour 12 patients, les autres ayant été perdus de vue sitôt après l'intervention. Le recul moyen était de 1 an.

\section{RÉSULTATS}

Nous avons colligé 15 cas d'adénome pléomorphe de localisation extra-parotidienne. Ils étaient répartis comme suit : 6 cas (40\%) au niveau de la glande submandibulaire, 5 cas (33\%) au niveau du palais dur, 3 cas $(20 \%)$ au niveau de la lèvre supérieure et 1 cas $(7 \%)$ à localisation para-pharyngée (figure 1). II s'agissait de 13 femmes et 2 hommes. L'âge moyen était de 48 ans avec des extrêmes allant de 38 à 70 ans. Aucun antécédent pathologique notable n'a été observé. Les patients ont consulté après un délai moyen de 4 ans avec des extrêmes allant de 2 mois à 10 ans.

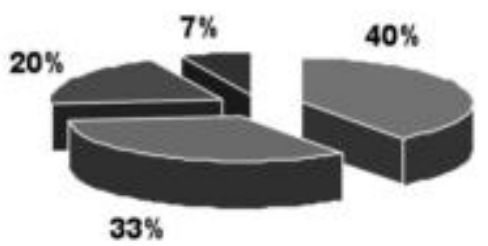

\begin{tabular}{l} 
- Glande sub_mandibulaire $=$ Palais \\
= Lèvre supérieure \\
\hline
\end{tabular}

\section{Fig. 1 : Siège des adénomes pléomorphes extra-parotidiens}

Le motif de consultation a été dans 14 cas (93\%) une tuméfaction isolée au niveau de la région intéressée, augmentant lentement de volume. Une dysphagie haute associée à une rhinolalie ont été rapportées par le patient ayant une tumeur à localisation para-pharyngée.

L'examen clinique retrouvait une tuméfaction ferme, mobile, indolore et bien limitée au niveau de la zone intéressée. La tumeur à localisation para-pharyngée a été responsable d'un bombement para-pharyngé droit refoulant l'amygdale en arrière.

L'échographie cervico-faciale a été pratiquée pour 5 cas de tumeurs à localisation submandibulaire. Elle identifiait le plus souvent une image bien limitée, hypoéchogène, hétérogène avec des logettes liquidiennes multiples. Dans 2 cas (40\%), l'échographie a été prise en défaut et a conclu à une adénopathie submandibulaire.

La cytoponction a été pratiquée dans 5 cas de tumeurs des glandes submandibulaires. Sa fiabilité était de $80 \%$ en orientant dans 4 cas vers un adénome pléomorphe. Dans 1 cas (20\%), elle a ramené un liquide séro-hématique et a été jugée non concluante. Deux localisations palatine ont bénéficié d'une biopsie préopératoire évo- quant le diagnostic d'adénome pléomorphe.

La tomodensitométrie a été pratiquée dans 6 cas. Quelque soit le siège de la tumeur, elle a montré une image bien limitée prenant le produit de contraste sans lyse osseuse en regard (figure 2).

L'imagerie par résonance magnétique a été pratiquée de première intention dans un cas à localisation submandibulaire, et en complément de la tomodensitométrie dans la localisation para-pharyngée. L'aspect retrouvé était une image bien limitée lobulée en hyposignal T1 et hypersignal T2 se rehaussant de manière homogène après injection de produit de contraste.

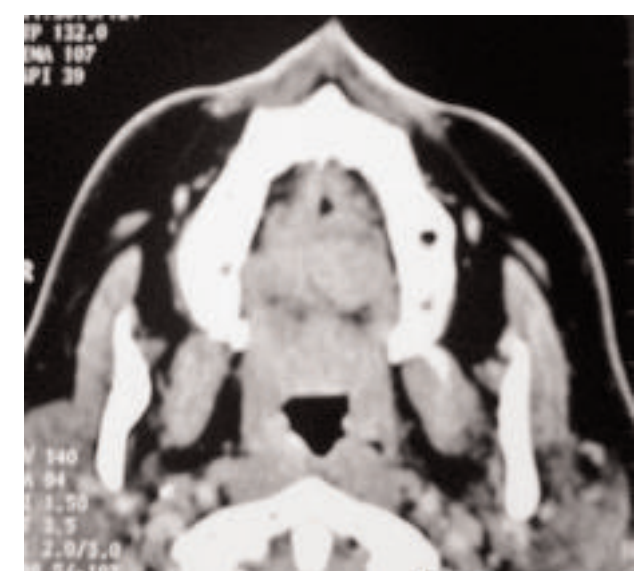

Fig. 2 : Tomodensitométrie du massif facial en coupe axiale : lésion du palais osseux prenant le contraste sans lyse osseuse en regard.

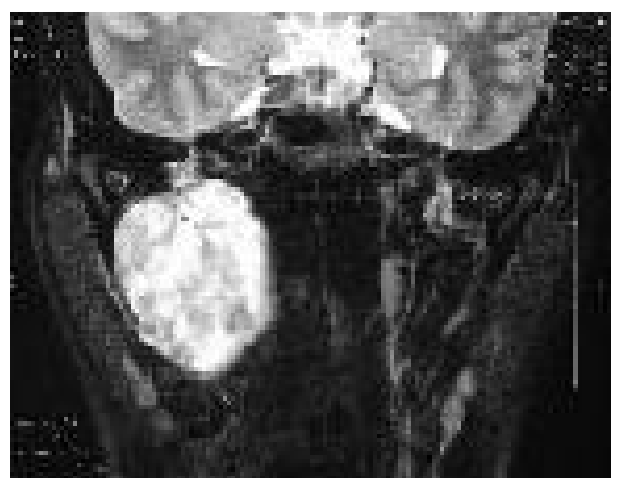

Fig. 3 : IRM en coupe coronale séquence T2 : tumeur parapharyngée

La chirurgie a été le traitement de choix pour tous les patients. Une exérèse sous anesthésie locale a été pratiquée dans les trois cas à localisation labiale. Une submandibulectomie par voie de Sébileau a été pratiquée pour les 6 cas à localisation submandibulaire. Les tumeurs à localisation palatines ont été opérées par voie endorale sous anesthésie générale. La tumeur de l'espace para-pharyngé a été également opérée par voie endorale pure. 
L'examen anatomo-pathologique extemporané a été réalisé dans 4 cas et avait une fiabilité de $75 \%$. Dans 1 cas, la nature histologique de la tumeur n'a pu être précisée par cet examen. L'examen anatomopathologique définitif de la pièce opératoire a confirmé le diagnostique d'adénome pléomorphe.

Après un recul moyen de un an, on n'a pas noté de récidives ni de transformation maligne.

\section{DISCUSSION}

L'adénome pléomorphe, ou tumeur mixte des glandes salivaires, représente 40 à $70 \%$ de toutes les tumeurs salivaires $(1,3,6)$. II se développe essentiellement au niveau de la parotide $(1,7)$. Dans notre série, il était de siège parotidien dans $65 \%$ des cas. Les localisations extra-parotidiennes représentaient $35 \%$ des cas. Elles étaient réparties entre la glande submandibulaire dans 14 $\%$ des cas et les glandes salivaires accessoires dans 21 $\%$ des cas. Ces données sont contraires à celles de la littérature qui montrent qu'en dehors de la glande parotide, la glande submandibulaire est le siège le plus fréquent des adénomes pléomorphes, suivi des glandes salivaires accessoires $(1,6)$.

Le palais renferme la plus grande concentration des glandes salivaires accessoires contenues dans le tractus aéro-digestif supérieur $(3,8)$. II est de ce fait le site le plus commun d'adénome pléomorphe des glandes salivaires accessoires, suivi de la lèvre supérieure, de la joue, du plancher buccal, du larynx et de la trachée $(2,9)$. Dans notre série, la majorité des tumeurs à développement intra-oral siégeaient également au niveau du palais et de la lèvre supérieure. D'autres localisations plus rares d'adénome pléomorphe des glandes salivaires accessoires ont été rapportées dans la littérature comme le septum nasal, le vestibule narinaire, le conduit auditif externe, la base de la langue, l'épiglotte, et l'espace parapharyngé $(5,7,10,11)$.

L'âge moyen de découverte des adénomes pléomorphes rapporté dans la littérature varie entre 36 et 47 ans $(1,6)$. Dans notre série, il était de 48 ans. Plusieurs études font état d'une prédominance féminine que nous avons retrouvée $(3,12)$.

Quelque soit son siège, l'adénome pléomorphe évolue habituellement selon un mode très lent et paucisymptomatique ; il est souvent découvert après quelques années d'évolution, sous la forme d'une tuméfaction isolée de la région intéressée $(1,2,13)$.

L'imagerie est indispensable dans le bilan pré-opératoire de l'adénome pléomorphe. Elle repose essentiellement sur la tomodensitométrie et l'imagerie par résonance magnétique. Elle permet de caractériser la tumeur, d'évaluer son extension aux tissus avoisinants et de rechercher une lyse osseuse $(3,8)$. L'imagerie par résonance magnétique est l'examen le plus performant pour l'exploration des tumeurs des glandes salivaires $(3,14)$. L'aspect typique d'un adénome pléomorphe est une tumeur lobulée, bien limitée, en hyposignal T1 et hypersignal T2 se rehaussant de manière homogène après injection de produit de contraste (14). L'imagerie par résonance magnétique permet aussi de différencier les tumeurs du lobe profond de la parotide à extension para-pharyngée de celles des glandes salivaires accessoires contenues dans l'espace para-pharyngé, en montrant pour ces dernières un liseré graisseux qui sépare la tumeur du parenchyme parotidien $(11,14,15)$.

La ponction cytologique est intéressante dans l'étude des adénomes pléomorphes de la glande submandibulaire avec une fiabilité de $90 \%(1,16)$. Elle a cependant quelques limites ; elle peut méconnaître un foyer de malignité au sein d'un adénome pléomorphe. Le diagnostic différentiel cytologique se pose classiquement avec le carcinome adénoïde kystique $(1,16)$.

Le traitement de l'adénome pléomorphe est chirurgical quelque soit sa localisation. L'exérèse peut se faire sous anesthésie locale ou générale $(2,4,13)$. Les tumeurs à localisation labiale sont souvent opérées sous anesthésie locale. La submandibulectomie sous anésthésie générale est indiquée pour les tumeurs de la glande submandibulire. Si les voies d'abord conventionnelles restent largement utilisées, Guerrissi et Taborda ont rapporté deux submandibulectomies endoscopiques par voie endorale (17). Les tumeurs du palais doivent bénéficier d'une exérèse complète par voie endorale $(3,9)$. Les tumeurs à localisation para-pharyngée peuvent être opérées par voies endorale, cervicale ou mixte $(11,15)$. Quelque soit la voie d'abord adoptée, l'exérèse de la tumeur doit se faire en totalité en évitant la rupture capsulaire qui est source de récidive $(11,15,18)$.

Sur le plan anatomo-pathologique, l'adénome pléomorphe des glandes salivaires accessoires est connu par l'importance de la cellularité avec peu d'éléments mésenchymateux $(4,19)$. La tumeur est généralement entourée d'une capsule fine ou incomplète expliquant les récidives tumorales $(5,20)$. Ces récidives sont décrites dans $2 \%$ des cas après chirurgie réglée et dans plus de $20 \%$ des cas après énucléation ; elles imposent donc de proscrire formellement toute énucléation ou chirurgie partielle en matière d'adénome pléomorphe $(1,19,20)$. Dans notre série, on n'a pas noté de récidive ni de transformation maligne . Celle-ci est de 9,1\% dans l'étude menée par Oueslati (1). Elle peut être synchrone ou survenir sur des récidives, ce qui impose une surveillance prolongée des patients opérés $(12,18)$.

\section{CONCLUSION}

L'adénome pléomorphe est la tumeur la plus fréquente des glandes salivaires avec une prédilection pour la parotide. Ses localisations extra-parotidiennes sont dominées par la glande submandibulaire et les glandes salivaires accessoires du palais et des lèvres. Chaque localisation 
est distincte par ses particularités cliniques, par les moyens de diagnostic et par la prise en charge chirurgicale.

\section{REFERENCES}

1- Oueslati Z, Naji R et al. Adénome pléomorphe de la glande submandibulaire. J Tun ORL $2003 ; 11: 19-22$.

2- Simon M. et al. Pleomorphic adenoma of a molar salivary gland. Oral Oncology extra $2005 ; 42$ : 170-2.

3- Lowry TR, Heichel DJ. Pleomorphic adenoma of the hard palate. Otolaryngol Head Neck Surg $2004 ; 131: 793$.

4- El Kohen A, Essakali L. et al. Adénome pléomorphe de la base de la langue. Rev Stomatol Chir Maxillofac $2007 ; 108: 215-17$.

5 - Maamouri M, B Hamouda R. et al. Adénome pléomorphe du septum nasal à propos d'un cas. J Tun ORL $2008 ; 21: 68-72$.

6- Alves FA, Perez DE, Lopes MA, Kowalski LP. Pleomorphic adenoma of the submandibular gland. Clinicopathological and immunohistochemichal features of 60 cases in Brazil. Arch Otolaryngol Head Neck Surg $2002 ; 128: 1400-3$.

7- Baek BJ, Bae CH, Kang EG, Cho HD. Pleomorphic adenoma of the nasal vestibile. Otolaryngol Head Neck Surg 2009 ; 141:535-6

8- Beckhardt RN, Weber RS, Zane R, et al. Minor salivary gland tumors of the palate :clinical and pathologic correlates of outcome. Laryngoscope 1995;105(11):1155-60

9- Lomeo P, Finneman J. Pleomorphic adenoma of the soft palate. Otolaryngol Head neck Surg 2001;125:122

10-Kumagai M, Endo S, Koizumi F, Kida A, Yamoto M. A case of pleomorphic adenoma of the nasal septum. Auris Nasus Larynx 2004;31:439-42

11- Ruis-Laza L, Infante-Cossio P, Garcia-Perla A, Hernandez-Guisalo JM, Gutierrez-Perez JL. Giant pleomorphic adenoma in the parapharyngeal space: report of 2 cases. J Oral Maxillofac Surg 2006;64:519-23.
12- Cwalina P, Skorek A, Narozny W, Stankiewicz C. Pleomorphic adenoma of minor salivary glands. Otolaryngol Pol 2002;56:737-40

13- Bablani D, Bansal S, Shetty SJ, Desai R, Kulkarni SR, Prasad P, Kayodkar FR. Pleomorphic adenoma of the cheek: a case report and review. J Oral Maxillofac Surg 2009; 67:1539-42

14- Bonfils P. Tumeurs des glandes salivaires. EMC ORL 2007;20-628-B-10

15- Morita N, Miyata K, Sakamoto T, Wada T. Pleomorphic adenoma in the parapharyngeal space. J Oral Maxillofac Surg 1995;53:605-10

16- Verma K, Kapila K. Role of fine needle aspiration cytology in diagnosis of peomorphic adenomas. Cytopathology 2002;13:121-7

17- Guerrissi JO, Taborda G. Endoscopic excision of the submandibular gland by an intraoral approach. J Craniofac Surg 2001;12:299-303

18- Ayoub OM, Bhatia K, Mal RK. Pleomorphic adenoma of the parotid gland: is long term follow-up needed? Auris Nasus Larynx 2002;29:283-5

19- Lopes MA, Kowalski LP, Santos GC, Almeida OP. A clinicopathologic study of 196 intraoral salivary gland tumors. J Oral Pathol Med 1999; 28: 264-7

20- Bentz BG, Hughes CA, Ludemann JP, Maddalozzo J. Masses of the salivary gland region in children. Arch Otolaryngol Head Neck Surg 2000; 126: 1435- 